\title{
XLVII. On quaternions; or on a new system of imaginaries in algebra
}

\author{
Sir William Rowan Hamilton LL.D. M.R.I.A. F.R.A.S.
}

To cite this article: Sir William Rowan Hamilton LL.D. M.R.I.A. F.R.A.S. (1849) XLVII. On quaternions; or on a new system of imaginaries in algebra, Philosophical Magazine Series 3, 34:230, 340-343, DOI: $10.1080 / 14786444908562659$

To link to this article: http://dx.doi.org/10.1080/14786444908562659

曲 Published online: 30 Apr 2009.

Submit your article to this journal $\sqsubset \pi$

Џ Article views: 3

Q View related articles $₫$

4 Citing articles: 1 View citing articles 
Experiment to separate Phosphoric from Pyrophosphoric Acid.

The different reaction of the phosphate and pyrophosphate of soda towards a solution of sulphate of magnesia and ammonia, led me to hope that it might form the basis of a method of separating these two modifications of phosphoric acid.

When pyrophosphate of soda is dissolved in a large amount of water, and the solution is mixed with a very large quantity of chloride of ammonium, no precipitate is produced on the addition of sulphate of magnesia and solution of ammonia. But at the end of a considerable period a precipitate falls, and is deposited firmly upon the sides of the vessel. If, however, the quantity of chloride of ammonium is very considerable, it frequently does not appear for several days.

$1.828 \mathrm{grm}$. of hydrated $c$-phosphate of soda, which had lost a small quantity of its water of crystallization by efflorescence, was dissolved in water with $1.521 \mathrm{grm}$. of the same salt, which had been previously heared to redness and furnished 0.611 grm. of pyrophosphate of soda. The solution was mixed with 100 grms. of chloride of ammonium, then diluted with 1600 grms. of water, and sulphate of magnesia and solution of ammonia added. The precipitate was filtored off after an interval of two hours, then washed, first with water containing chloride of ammonium, afterwards with water containing ammonia. 0.814 grm. of calcined phosphate of magnesia was obtained, which contains $0.516 \mathrm{grm}$. of phosphoric acid. But the 1.828 grm. of phosphate of soda contains only $0.391 \mathrm{grm}$. of phosphoric acid : hence a considerable amount of pyrophosphoric acid was precipitated with the phosphate of annionia and magnesia. This method of separation is consequently inapplicable.

XLVII. On Quaternions; or on a New System of Imaginaries in Algebra. By Sir Wrimam Rowan Hamuton, LL.D., M.R.I.A., F.R.A.S., Corresponding Member of the Institute of France, \&c., Andreres' Professor of Astronomy in the University of Dublin, and Royal Astronomer of Ireland.

[Continued from p. 297.]

68. TTHE equation of the ellipsoid (see Philosophical 1 Magazine for October 1847, or Proceedings of the Royal Irish Academy for July 184.6),

$$
T(i p+p x)=x^{2}-i^{2} \text {, eq. (9.), art. } 38 \text {, }
$$

which has so often presented itself in these researches, may be anew transformed as follows. Writing it thus,

$$
\mathrm{T} \frac{(\mathfrak{p}+p x)(1-x)}{x^{2}-1^{2}}=\mathrm{T}(1-x), \ldots .
$$


which we are allowed to do, because the tensor of a product is equal to the product of the tensors, we may observe that while the denominator of the fraction in the first member is a pure scalar, the numerator is a pure vector; for the identity,

gives

$$
p+p x=S .(b+x) p+V .(b-x) p, . .
$$

$$
\text { S. }(p+p x)(1-x)=0 \text { : . }
$$

the fraction itself is therefore a pure vector, and the sign $T$, of the operation of taking the tensor of a quaternion, may be changed to the sign TV, of the generally distinct but in this case equivalent operation, of taking the tensor of the vector part. But, under the sign $\mathrm{V}$, we may reverse the order of any odd number of vector factors (see article 20 in the Philosophicat Magazine for July 1846); and thus may change, in the numerator of the fraction in (125.), the partial product $p(a-x)$ to $(1-x) p$. Again, it is always allowed to divide (though not, generally, in this calculus, to multiply) both the numerator and denominator of a quaternion fraction, by any common quaternion, or by any common vector; that is, to multiply beth numerator and denominator into the reciprocal of such common quaternion or vector: namely by writing the symbol of this new factor to the right (but not generally to the left) of both the symbols of numerator and denominator, above and below the fractional bar. Dividing therefore thus above and below by 1 , or multiplying into $t^{-1}$, after that permitted transposition of factors which was just now specified, and after the change of $\mathrm{T}$ to $\mathrm{TV}$, we find that the equation (125.) of the ellipsoid assumes the following form:

$$
T V \frac{(1-x) p+p\left(x-x^{2}-1\right)}{(1-x)+\left(x-x^{2} t^{-1}\right)}=T(1-x) ; \quad . \quad .
$$

the new denominator first presenting itself under the form $x^{2} t^{-1}-\imath$, but being changed for greater symmetry to that written in (128.), which it is allowed to do, because, under the sign $T$, or under the sign $T V$, we may multiply by negative unity.

69. In the last equation of the ellipsoid, since

we have

$$
x-x^{2} b^{-1}=x(1-x) t^{-1} \text {, }
$$

$$
\mathrm{T}\left(x-x^{2} t^{-1}\right)=\mathrm{T} x \mathrm{~T}(1-x) \mathrm{T}^{-1} ; \quad .
$$

and under the characteristic $U$, of the operation of taking the versor of a quaternion, w'e may multiply by any positive scalar, such as $-x^{-2}$ is, because $x^{2}$ and $x^{-2}$ are negative*

- By this, which is one of the earliest and most fundanental principles of the whole quaternion theory (see the author's letter to John T. Graves, 
scalars; whereas to multiply by a negative scalar, under the same sign $\mathrm{U}$, is equivalent to multiplying the versor itself by -1 : hence,

$$
\mathrm{U}\left(x-x^{2}-1\right)=-\mathrm{U}\left(x^{2} b^{-1}-x\right)=-\mathrm{U}\left(x^{-1}-1^{-1}\right) .
$$

If then we introduce two new fixed vectors, $\eta$ and $\theta$, defined by the equations,

$$
\eta=\mathrm{T}_{1} \mathrm{U}(b-x) ; \theta=\mathrm{T}_{x} \mathrm{U}\left(x^{-1}-t^{-1}\right) ; .
$$

and if we remember that any quaternion is equal to the product of its own tensor and versor (Phil. Mag. for July 1846); we shall obtain the transformations,

$$
1-x=\eta \mathrm{T} \frac{1-x}{1} ; x-x^{2} 1^{-1}=-\theta \mathrm{T} \frac{1-x}{1} ; .
$$

which will change the equation of the ellipsoid (128.) to the following :

$$
\mathrm{TV} \frac{\eta p-\rho^{\theta}}{\eta-\theta}=\mathrm{T}(b-x) . \quad . \quad . .
$$

70. To complete the elimination of the two old fixed vectors, $1, x$, and the introduction, in their stead, of the two new fixed vectors, $\eta, \theta$, we may observe that the two equations (132.) give, by addition,

$$
1-x^{2}-1=(y-\theta) T^{1} \frac{-x}{b} ; . . . .
$$

taking then the tensors of both members, dividing by $\mathrm{T}^{1-x}$, and attending to the expression (81.) in article 56, (Phil. Mag. for May 1848, for the mean semiaxis $b$ of the ellipsoid, we find this new expression for that semiaxis:

$$
\mathrm{T}(\eta-\theta)=\frac{x^{2}-t^{2}}{\mathrm{~T}(t-x)}=b . \quad . \quad . \quad .
$$

Esq., of October 17th, 1843, printed in the Supplementary Number of the Philosophical Magazine for December 1844), namely by the principle that the square of EVERY VECTOR (or directed straight line in tridimensional space) is to to be regarded as a NEGATIVE NUMBER, this theory is not merely distinguished from, but sharply CONTRASTED with, every other sysiem of algebraic geometry of which the present writer has hitherto acquired any knowledge, or received any intimation. In saying this, he hopes that he will not be supposed to desire to depreciate the labours of any other past or present inquirer into the properties of that important and precious Symbol in Geometry, $\sqrt{-1}$. And he gladly takes occasion to repeat the expression of his sense of the assistance which he received, in the progress of his own speculations, from the study of Mr. IVarren's work, before he was able to examine any of those earlier essays referred to in Dr. Peacock's Report: however disinct, and even contrasted, on several fundamental points, may be (as was above observed) the methods of the CaLculus of Quaternions from those of what Professor De Morgan has happily named Double ALGEBRA. 
But also, by (131.), or by (132.),

and therefore,

$$
\mathrm{T}_{\eta}=\mathrm{T}_{t} ; \mathrm{T} \theta=\mathrm{T}_{x} ; \ldots . .
$$

$$
\theta^{2}-\eta^{2}=x^{2}-t^{2} . \quad . \quad . \quad . \quad
$$

Hence, by (135.), we obtain the expression,

$$
\mathbf{T}(1-x)=\frac{\theta^{2}-\eta^{2}}{\mathbf{T}(\eta-\theta)} ; \quad . \quad . \quad .
$$

which may be substituted for the second member of the equation (133.), so as to complete the required elimination of $t$ and $x$. And if we then multiply on both sides by $T(\eta-\theta)$, n'e obtain this new form* of the equation of the ellipsoid:

$$
\mathrm{TV} \frac{\eta p-p^{\theta}}{\mathrm{U}(\eta-\theta)}=\theta^{2}-\eta^{2} ; \quad . \quad . \quad .
$$

which will be found to include several interesting geometrical significations.

- This form was conmunicated to the Royal Irish Academy, at the stated meeting of that body on March 16th, 1849, in a note addressed by the present writer to the Rev. Charles Graves. It was remarked, in that note, that the directions of the two fixed vectors $\eta, \theta$, are those of the two asymptotes to the focal hyperbola; while their lengths are such that the two extreme semiaxes of the ellipsoid may be expressed as follows :

$$
a=\mathrm{T} n+\mathrm{T} \theta ; c=\mathrm{T} n-\mathrm{T} \theta ;
$$

the mean semiaxis being, at the same time, expressed (as in the text of the present paper) by the formula

$$
b=T(n-\theta) \text {. }
$$

It was observed, further, that $n-\theta$ has the direction of one cyclic normal of the ellipsoid, and that $n^{-1}-\theta^{-1}$ has the direction of the other cyclic normal; that $n+\theta$ is the vector of one umbilic, and that $x^{-1}+\theta^{-1}$ has the direction of another umbilicar vector, or umbilicar semidiameter of the ellipsoid; that the focal ellipse is represented by the system of the two equations

and

$$
\mathrm{S} . \rho \mathrm{U} \mathrm{U}_{\eta}=\mathrm{S} . \rho \mathrm{U} \theta \text {, }
$$

$$
\text { TV. } U_{n}=2 S \sqrt{n \bar{\theta}},
$$

of which the first represents its plane, while the second, which (it was remarked) might also be thus written,

$$
\text { TV. } \mathrm{\rho} T \theta=2 \mathrm{~S} \sqrt{n \theta},
$$

represents a cylinder of revolution (or, under the latter form, a second cylinder of the same kind), whereon the focal ellipse is situated; and that the focal hyperbola is adequately expressed or represented by the single equation,

$$
\mathrm{V} \cdot \eta_{\mathrm{g}} \cdot \mathrm{V} . \rho \theta=(\mathrm{V} . \eta \theta)^{2} \text {. }
$$

To which it may be added, that by changing the two fixed vectors $n$ and $\theta$ to others of the forms $t^{-1} n$ and $t \theta$, we pass to a confocal surface.

[To be continued.] 